\title{
CONTAMINAÇÃO MICROBIOLÓGICA EM PLANTAS MEDICINAIS E HORTALIÇAS E SUA IMPLICAÇÃO NO ESTADO DE SAÚDE DO CONSUMIDOR: REVISÃO
}

\begin{abstract}
Mardjori Andrade Hellmann
Leonardo Garcia Velasquez ${ }^{2}$

HELLMANN, M. A.; VELASQUEZ, L. G. Contaminação microbiológica em plantas medicinais e hortaliças e sua implicação no estado de saúde do consumidor: revisão. Arq. Cienc. Saúde UNIPAR, Umuarama, v. 21, n. 2, p, 123-130, maio/ago. 2017.

RESUMO: A utilização de plantas medicinais está em continuo crescimento, entretanto alguns quesitos de qualidade precisam ser avaliados, como a contaminação microbiológica e parasitológica da matéria prima que pode apresentar um impacto importante no estado de saúde do consumidor. O presente estudo teve por objetivo identificar a ocorrência de contaminação microbiológica e parasitológica em plantas medicinais, foram analisados artigos publicados nos últimos 20 anos nas bases eletrônicas de dados LILACS, PubMed e SciELO, utilizando os descritores "microbiológico", "contaminação", "plantas medicinais", "hortaliças", "parasito" e seus correspondentes em inglês. Foram selecionados 9 artigos que abordam o tema contaminação em plantas medicinais e 15 artigos sobre contaminação em hortaliças, totalizando 24 artigos. O potencial de contaminação por parasitos em plantas medicinais e hortaliças é semelhante devido à forma de cultivo e consumo de ambas. Foram encontrados ovos de Ancylostoma sp., Ascaris sp., Taenia sp., os fungos Aspergillus sp., Penicillium sp., Fusarium sp., e em um estudo Salmonella sp. Não foram encontrados, nas bases pesquisadas, trabalhos que avaliaram a contaminação por parasitos em plantas medicinais, salienta-se a necessidade de fiscalização, a fim de melhorar a qualidade higiênico-sanitária das plantas medicinais e hortaliças ofertadas à população.
\end{abstract}

PALAVRAS-CHAVE: Análise microbiológica. Contaminação. Hortaliças. Parasitos. Plantas Medicinas.

\section{MICROBIOLOGICAL CONTAMINATION IN MEDICINAL PLANTS AND VEGETABLES AND THE IMPLICATIONS ON CONSUMER HEALTH: REVIEW}

\begin{abstract}
The use of medicinal plants is continuously increasing. However, some quality requirements need to be assessed, such as the microbiological and parasitological contamination of raw material, which can have an important impact on the health of the consumers. This study aimed to identify the occurrence of microbiological and parasitological contamination in medicinal plants, and in order to do so, studies published in the last 20 years in the LILACS, PubMed and SciELO electronic databases were analyzed, using the terms "microbiological", "contamination", "medicinal plants ", "vegetable", "parasite" and their Portuguese correspondents. A total of 24 articles were reviewed, with 9 addressing contamination in medicinal plants and 15 addressing contamination in vegetables. The potential for parasite contamination in medicinal plants and vegetables is similar due to the way they are cultivated and consumed. Eggs from Ancylostoma sp., Ascaris sp., Taenia sp., Fungi Aspergillus sp., Penicillium sp., Fusarium sp., and, in one study, from Salmonella sp were reported. In the analyzed databases, there were no studies assessing the contamination by parasites in medicinal plants. Therefore, further studies are necessary in order to improve the hygienic-sanitary quality of the medicinal plants and vegetables offered to the population.
\end{abstract}

KEYWORDS: Contamination. Medicinal plants. Microbiological analysis. Parasites. Vegetables.

\section{Introdução}

A utilização de plantas medicinais está em continuo crescimento e a busca por agentes terapêuticos derivados de espécies vegetais, frente a uma oferta insuficiente de plantas medicinais, levou a uma queda na sua qualidade. As plantas medicinais são alternativas terapêuticas importantes, entretanto alguns quesitos precisam ser avaliados, como a contaminação microbiológica e parasitológica da matéria prima que pode apresentar um impacto importante no estado de saúde do consumidor (SOUZA; LIONZO; PETROVICK, 2006).

Aproximadamente $20 \%$ da população brasileira consome $63 \%$ dos medicamentos alopáticos sintéticos enquanto $60 \%$ faz uso de plantas medicinais como fonte alternativa de tratamento. As plantas são utilizadas principalmente para tratar afecções renais, reumatismo, diabetes, processos inflamatórios, dores estomacais, como adjuvantes no processo de cicatrização, doenças respiratórias entre outras e a principal forma de utilização é por meio de chás, em decocto ou infuso (SOUSA, 2014).

Plantas medicinais, nativas ou cultivadas, são suscetíveis ao ataque por ácaros, nematoides e fungos e o controle dessas pragas é um desafio ao produtor, pois não existem pesticidas registrados para o uso em plantas medicinais. Técnicas como a rotação de culturas, fertilização e irrigação apropriadas e a inserção de plantas aromáticas, de forma alternada com hortaliças e espécies frutíferas, que atuem como atraentes de inimigos naturais dessas pragas ou como repelentes, também podem auxiliar na prevenção de doenças compondo um sistema agroflorestal que dificulta a proliferação dessas pragas (BEVILAQUA et al., 2012; CARVALHO et al., 2010).

Durante toda a cadeia produtiva, desde o plantio, colheita, processamento, armazenamento e transporte das plantas medicinais, deixam-as suscetíveis à contaminação por uma variedade de resíduos e microrganismos, em parte por esses processos serem realizados, na maioria das vezes, sem os devidos cuidados de higiene e controle sanitário. Além de muitos produtores desconhecerem os cuidados ne-

DOI: https://doi.org/10.25110/arqsaude.v21i2.2017.5862

${ }^{1}$ Professora da Universidade Paranaense - UNIPAR, Francisco Beltrão e Mestranda do Programa em Plantas Medicinais e Fitoterápicos na Atenção Básica, UNIPAR, Umuarama. Travessa dos Bancários, 45. Vila Nova, Francisco Beltrão -PR. 85601-000. Email: mardjori@gmail.com

${ }^{2}$ Professor da Universidade Paranaense UNIPAR - Francisco Beltrão. Rua Guanabara, 365 - apartamento 303. cep: 85605-300. Presidente Kennedy, Francisco Beltrão - PR. email: leo@prof.unipar.br 
cessários para garantir as boas práticas de produção, as características biológicas da própria planta e o elevado teor de umidade favorecem a contaminação e o crescimento microbiano (SOUZA; LIONZO; PETROVICK, 2006).

Falhas no controle de qualidade da matéria-prima e insumos ao longo da cadeia produtiva podem permitir a veiculação de uma microbiota, por vezes patogênica, e esses microrganismos, quando presentes em níveis acima do preconizado em plantas medicinais utilizadas para o preparo de chás, podem gerar problemas à saúde dos consumidores. A decocção, processo tradicional de preparo, em que partes da planta são colocadas em contato com a água em ebulição, pode gerar produtos com reduzida carga microbiana, entretanto, dependendo do grau de contaminação na matéria-prima inicial, esse processo pode não ser efetivo (SOUZA; LIONZO; PETROVICK, 2006).

Com a crescente busca por produtos naturais e seus derivados uma avaliação mais criteriosa da qualidade dos produtos oferecidos ao consumidor deve ser realizada, a partir da legislação específica, controlando todos os estágios da produção. O controle e a avaliação da carga microbiológica e parasitológica das plantas medicinais são determinantes para a garantia da sa qualidade e caso não sejam adequadamente cumpridos podem comprometer a utilização destas como adjuvantes na melhoria do estado de saúde do indivíduo. A falta de padronização durante as etapas da produção, a baixa qualidade, a adulteração do material e a utilização de forma inapropriada desses produtos comprometem a sua eficácia terapêutica, além de acarretarem riscos à saúde do consumidor (CARVALHO et al., 2010; COSSATIS, 2015; RATAJCZAK et al., 2015; SOUZA; LIONZO; PETROVICK, 2006).

Desde 2006, o Brasil possuiu políticas públicas com a finalidade de fomentar o uso de plantas medicinais como a Política Nacional de Plantas Medicinais e Fitoterápicos, por meio do decreto $\mathrm{n}^{\circ} 5.813$, de 22 de junho de 2006, que estipula a inserção de terapias alternativas e práticas populares no sistema único de saúde (SUS) juntamente com a relação nacional de plantas medicinais de interesse ao SUS (RENISUS) que fazem parte das políticas públicas de saúde, meio ambiente, desenvolvimento econômico e social, que estimulam ações capazes de promover melhorias na qualidade de vida da população (BEZERRA CARVALHO et al., 2014; BOCHNER et al., 2012; BRASIL. MINISTÉRIO DA SAÚDE, 2006; LANINI et al., 2009).

Plantas medicinais e as hortaliças em geral, devido a sua forma de cultivo, armazenamento, comercialização e transporte, podem representar importantes veículos de transmissão de enteropatógenos para hospedeiros susceptíveis, considerando sua origem natural e a forma de consumo de ambas, podendo desencadear no consumidor desde infecções assintomáticas até quadros mais graves com síndrome da má absorção, emagrecimento, desenvolvimento de anemias, quadros de diarreia, oclusão, perfuração intestinal e dores abdominais (ANDRADE et al., 2010; SCHEFFER et al., 2006).

Com o interesse cada vez maior da população por terapias naturais e a demanda aumentada por produtos à base de plantas medicinais a presente pesquisa teve por objetivo fazer uma revisão bibliográfica, a fim de verificar se esses produtos estão sendo oferecidos ao consumidor de acordo com a legislação específica no quesito contaminação microbiológica e parasitológica.

\section{Metodologia}

O presente estudo trata-se de uma análise de dados secundários, por meio de uma revisão da literatura sobre contaminação microbiológica e parasitológica em plantas medicinais e hortaliças e sua implicação no estado de saúde do consumidor.

Para tanto, foram analisados artigos científicos publicados nos últimos 20 anos, as buscas foram realizadas nas bases eletrônicas de dados LILACS, PubMed e SciELO, utilizando os descritores previamente consultados no DECs (Descritores em Ciências da Saúde) em português "plantas medicinais", "contaminação", "hortaliças" e "parasito" e seus correspondentes em Inglês. Foram incluídos no estudo os artigos que mencionavam algum tipo de contaminação microbiológica ou parasitológica em plantas medicinais e hortaliças e suas implicações no estado de saúde e excluídos os trabalhos que abordavam outras formas de contaminação, que não a microbiológica ou parasitológica, aqueles que avaliavam a atividade das plantas medicinais contras agentes microbiológicos ou parasitológicos ou que estivessem redigidos em outras línguas que não português e inglês.

A análise dos artigos foi realizada em três etapas. A primeira foi avaliar os textos quanto ao título, na segunda etapa, foi realizada a leitura dos resumos dos artigos selecionados na primeira fase da avaliação, foram selecionados os que mencionavam algum tipo de contaminação microbiológica ou parasitológica em plantas medicinais e hortaliças. Na sequência foi feita a avaliação integral dos artigos escolhidos na fase da leitura dos resumos, a fim de selecionar os que citavam as possíveis implicações no estado de saúde do consumidor bem como os principais contaminantes. As informações encontradas nos artigos foram sistematizadas e descritas.

\section{Resultados}

A tabela 1 mostra os resultados encontrados em cada uma das bases de dados pesquisadas, utilizando os descritores "plantas medicinais" e "contaminação". Dessa forma, foram selecionados 9 (nove) artigos de estudos científicos que abordam o tema contaminação em plantas medicinais para apresentação no presente trabalho, além disso 15 (quinze) artigos sobre contaminação em hortaliças foram acrescentados à pesquisa (Tabela 3), totalizando 24 artigos.

Tabela 1: Resultado das buscas nas bases PubMed, LILACS e SciELO utilizando os descritores "plantas medicinais" e "contaminação".

\begin{tabular}{lccc}
\hline $\begin{array}{c}\text { Base de } \\
\text { Dados }\end{array}$ & $\begin{array}{c}\text { Total de } \\
\text { Artigos }\end{array}$ & $\begin{array}{c}\text { Excluídos após } \\
\text { leitura de títulos e } \\
\text { resumos }\end{array}$ & Selecionados \\
\hline PubMed & 255 & 253 & 2 \\
LILACS & 31 & 27 & 4 \\
SciELO & 20 & 17 & 3 \\
Total & $\mathbf{3 0 6}$ & $\mathbf{2 9 7}$ & $\mathbf{9}$ \\
\hline
\end{tabular}

O estudo concentrou-se em 9 artigos de interesse (2,94\%) do total de artigos publicados nas três bases de dados). Analisando os dados da presente revisão, e enfatizando 
a Tabela 2, descrita abaixo, na maioria dos estudos a pesquisa de bactérias e fungos é realizada, não sendo encontrado nenhum estudo, nas bases de dados pesquisadas, que avaliasse a presença de parasitos em plantas medicinais, vários traba- lhos avaliaram a atividade antiparasitária da planta contra um patógeno específico, porém, não encontramos estudos avaliando a possível contaminação da planta por parasitos.

Tabela 2: Principais contaminantes encontrados em amostras de plantas medicinais.

\begin{tabular}{|c|c|c|c|c|}
\hline Autores & País & Planta(s) pesquisadas & Microrganismos Encontrados & $\begin{array}{l}\text { Modo de } \\
\text { utilização }\end{array}$ \\
\hline (SANTOS et al., 2013) & Brasil & $\begin{array}{l}\text { Peumus boldus } \\
\text { Pimpinella anisum } \mathrm{L} . \\
\text { Matricaria chamomilla } \mathrm{L} .\end{array}$ & $\begin{array}{l}\text { Aspergillus sp., Penicillium sp. e Fusarium sp., } \\
\text { Exophiala sp. e Fonsecaea sp., }\end{array}$ & Infuso \\
\hline (ZARONI et al., 2004) & Brasil & Plantas Medicinais & $\begin{array}{l}\text { Escherichia coli, }(22,22 \%) \\
\text { Pseudomonas aeruginosa, }(23,61 \%)\end{array}$ & Infuso \\
\hline $\begin{array}{l}\text { (MARCONDES; } \\
\text { ESMERINO, 2010) }\end{array}$ & Brasil & $\begin{array}{l}\text { Plantas medicinais } \\
\text { provenientes das hortas } \\
\text { domésticas }\end{array}$ & Coliformes fecais $(27,3 \%)$ & Infuso \\
\hline (SILVA et al., 2012) & Brasil & Plantas Medicinais & $\begin{array}{l}\text { Fungos filamentosos e leveduriformes }(72,3 \%) \\
\text { E. coli }(4 / 10) \\
\text { Salmonella } \text { sp. }(2 / 10)\end{array}$ & Infuso \\
\hline (MAXIMINO et al., 2011) & Brasil & Chamomilla recutita $\mathrm{L}$. & $\begin{array}{l}\text { Aspergillus spp. } \\
\text { Penicillium spp. }\end{array}$ & Infuso \\
\hline (YESUF et al., 2016) & Etiópia & Plantas Medicinais & $\begin{array}{l}\text { Bacillus }(20.7 \%) \\
\text { Enterobacter cloacae }(12 \%) \\
\text { Shigella dysenteriae }(8.7 \%) \\
\text { Citrobacter spp. }(8 \%) \\
\text { Providencia } \text { spp. }(8 \%) \\
\text { Klebsiella pneumoniae }(7.3 \%) \\
\text { Escherichia coli }(6.7 \%) \\
\text { Staphylococcus aureus }(6.7 \%) \\
\text { Staphylococcus epidermidis }(3.3 \%) \\
\end{array}$ & Infuso \\
\hline (CARVALHO et al., 2010) & Brasil & $\begin{array}{l}\text { Chamomilla recutita } \mathrm{L} . \\
\text { Pimpinella anisum } \mathrm{L} . \\
\text { Ilex paraguariensis } \text { St.-Hil. }\end{array}$ & $\begin{array}{l}\text { Aspergillus sp. }(35,9 \%) \\
\text { Penicillium sp. }(9,4 \%) \\
\text { Fusarium sp. }(0,21 \%) \\
\text { Rhizopus sp. }(11,5 \%) \\
\text { Ulocladium sp. }(18,4 \%) \\
\text { Mycelia sterilia }(6,84 \%)\end{array}$ & Infuso \\
\hline (MELO et al., 2007) & Brasil & Capim-limão & $\begin{array}{l}\text { Coliformes a } 35^{\circ} \mathrm{C}(50 \%) \\
\text { Coliformes a } 45^{\circ} \mathrm{C}(3 / 16) \\
\text { Escherichia coli }(1 / 16) \\
\text { Bolores e leveduras }(81,25 \%)\end{array}$ & Infuso \\
\hline
\end{tabular}

Microrganismos identificados em apenas 1 estudo foram Enterobacter cloacae, Shigella dysenteriae, Citrobacter spp., Providencia spp., Klebsiella pneumoniae, Staphylo-

coccus aureus, Staphylococcus epidermidis, Pseudomonas aeruginosa e os fungos, Exophiala sp. e Fonsecaea sp.

Tabela 3: Resultado das buscas nas bases PubMed, LILACS e SciELO utilizando os descritores "parasitos" e "hortaliças".

\begin{tabular}{lccc}
\hline \multicolumn{1}{c}{ Base de Dados } & Total de Artigos & Excluídos após leitura de títulos e resumos & Selecionados \\
\hline PubMed & 249 & 244 & 5 \\
LILACS & 48 & 41 & 7 \\
SciELO & 14 & 11 & 3 \\
Total & 311 & 296 & 15 \\
\hline
\end{tabular}

O estudo concentrou-se em 15 artigos de interesse $(4,82 \%)$ do total de artigos publicados nas três bases de dados. 
Tabela 4: Principais contaminantes encontrados em amostras de hortaliças.

\begin{tabular}{|c|c|c|c|c|}
\hline Autores & País & $\begin{array}{c}\text { Microrganismos } \\
\text { Encontrados }\end{array}$ & Parasitos Encontrados & $\begin{array}{l}\text { Modo de } \\
\text { utilização }\end{array}$ \\
\hline (PAULA et al., 2003) & Brasil & $\begin{array}{c}\text { Mesófilos aeróbios } \\
(53,3 \%)\end{array}$ & Entamoeba coli $(9,9 \%)$ & In natura \\
\hline (COELHO et al., 2001) & Brasil & & $\begin{array}{l}\text { Strongyloides stercoralis e Ancilostomideos, }(83,3 \%) \\
\text { Giardia lamblia, }(9,5 \%) \\
\text { ovos de Ascaris lumbricoides }(4,8 \%) \\
\text { ovos de Ancilostomídeos }(2,4 \%)\end{array}$ & In natura \\
\hline (ROSTAMI et al., 2016) & Iran & & $\begin{array}{l}\text { Ascaris lumbricoides }(3.36 \%) \\
\text { Trichuris trichiura }(2.2 \%) \\
\text { hookworms }(2.9 \%) \\
\text { Toxocara spp. }(1.68 \%) \\
\text { Trichostrongylus } \mathrm{spp} .(1.55) \\
\text { Taenia } \text { spp. }(0.9 \%) \\
\text { Hymenolepis nana }(2.2 \%)\end{array}$ & In natura \\
\hline (DUEDU et al., 2014) & Ghana & & $\begin{array}{l}\text { Cryptosporidium parvum, } \\
\text { Entamoeba histolytica/dispar, } \\
\text { Giardia lamblia, } \\
\text { Cyclospora cayentenesis, } \\
\text { Isospora belli, } \\
\text { Entamoeba coli } \\
\text { Strongyloides stercoralis, } \\
\text { Trichuris trichiuria, } \\
\text { Eterobious vermicularis, } \\
\text { Faciolopsis buski }\end{array}$ & In natura \\
\hline (KŁAPEĆ; BORECKA, 2009) & Polônia & & $\begin{array}{l}\text { Toxocara spp. }(19.2 \%) \\
\text { Ascaris spp. }(5.4 \%) \\
\text { Trichuris spp. }(3.8 \%) \\
\end{array}$ & In natura \\
\hline (NOMURA et al., 2015) & Brasil & & $\begin{array}{l}\text { Endolimax nana }(100 \%) \\
\text { Larvas de Ancilostomideos }(87,5 \%) \\
\text { Cistos de Balantidium col }(87,5 \%) \\
\text { Entamoeba histolytica }(87,5 \%) \\
\text { Strongyloides stercoralis }(75 \%) \\
\text { Entamoeba coli }(62,5 \%) \\
\text { Ovos de Ascaris } \operatorname{spp} .(37,5 \%)\end{array}$ & In natura \\
\hline $\begin{array}{l}\text { (DA SILVA ALVES; } \\
\text { DA CUNHA NETO; } \\
\text { ROSSIGNOLI, 2013) }\end{array}$ & Brasil & & $\begin{array}{l}\text { Ascaris sp. }(36,9 \%) \\
\text { Ancilostomideos }(18,7 \%) \\
\text { Larvas de outros nematoides }(21,6 \%) \\
\text { Balantidium sp. }(12,6 \%) \\
\text { Cistos de Entamoeba } \mathrm{sp} .(5,5 \%)\end{array}$ & In natura \\
\hline $\begin{array}{l}\text { (GONÇALVES; DA SILVA; } \\
\text { STOBBE, 2013) }\end{array}$ & Brasil & & $\begin{array}{l}\text { Oocistos não esporulados }(28,8 \%) \\
\text { Larvas e adultos de nematódeos de vida livre }(46,6 \%)\end{array}$ & In natura \\
\hline $\begin{array}{l}\text { (PACIFICO; BASTOS; } \\
\text { UCHÔA, 2013) }\end{array}$ & Brasil & & $\begin{array}{l}\text { Oocisto não esporulado de coccídio ( } 4 \text { \%) } \\
\text { Cisto de amebídeo com menos de quatro núcleos ( } 2 \% \text { ) } \\
\text { Ovos de ancilostemídeos ( } 12 \%)\end{array}$ & In natura \\
\hline $\begin{array}{l}\text { (FRIAS; SILVA; TOZATO, } \\
\text { 2012) }\end{array}$ & Brasil & & $\begin{array}{l}\text { Almeirão } \\
\text { Ascaris sp. }(2,20 \%) \\
\text { Hymenolepis } \mathrm{sp} .(36,09 \%) \\
\text { Dipylidium } \mathrm{sp} .(15,98 \%) \text { Diphilobotrium sp. }(4,14 \%) \\
\text { Taenia } \mathrm{sp} .(3,58 \%) \\
\text { Ancylostoma } \mathrm{sp} .(2,20 \%) \\
\text { Agrião } \\
\text { Ascaris } \mathrm{sp} .(80,23 \%) \\
\text { Taenia } \mathrm{sp} .(6,60 \%) \\
\text { Dipylidium } \mathrm{sp} .(5,50 \%) \text { Diphilobotrium } \mathrm{sp} .(3,29 \%) \\
\text { Hymenolepis } \mathrm{sp} .(3,29 \%) \\
\text { Enterobius } \mathrm{sp} .(1,09 \%)\end{array}$ & In natura \\
\hline
\end{tabular}




\begin{tabular}{|c|c|c|c|}
\hline $\begin{array}{l}\text { (FERRO; COSTA-CRUZ; } \\
\text { BARCELOS, 2012) }\end{array}$ & Brasil & $\begin{array}{l}\text { Larvas rabditoides } \\
\text { de Strongyloides spp. } \\
\text { Ovos não larvados de Toxocara sp. } \\
\text { Ovos de Fasciola hepatica } \\
\text { Cistos de Entamoeba coli }\end{array}$ & In natura \\
\hline (BELINELO et al., 2009) & Brasil & $\begin{array}{l}\text { Larvas de Strongyloides spp. (4\%) } \\
\text { Ovos de Ascaris spp. (9\%) } \\
\text { Ancylostoma spp. }(4 \%) \\
\text { Trichuris spp. }(5 \%) \\
\text { Taenia spp. }(4 \%) \\
\text { Entamoeba histolytica }(28 \%) \\
\text { Giardia lamblia }(16 \%)\end{array}$ & In natura \\
\hline (SOARES; CANTOS, 2005) & Brasil & Enteroparasitos $(73,3 \%)$ & In natura \\
\hline (ARBOS et al., 2010) & Brasil & $\begin{array}{l}\text { Ovos de ancilostomídeo }(26,7 \%) \\
\text { Cistos de Entamoeba sp. }(20 \%)\end{array}$ & In natura \\
\hline
\end{tabular}

\section{Discussão}

Segundo Martinazzo (2006) entre as origens da má qualidade de plantas medicinais, encontram-se as contaminações microbiológicas (bactérias, fungos, etc) e físicas (solo, partículas, etc) associadas ao processo de secagem, transporte, utilização de embalagens inadequadas e a falta de práticas agrícolas adequadas. Uma vez colhida, a planta pode perder qualidade nas etapas seguintes do processamento o que torna os processos de secagem e armazenamento fundamentais para a qualidade final do produto.

A secagem visa a reduzir a ação enzimática por meio da diminuição do teor de umidade, pois os microrganismos, as enzimas e o próprio metabolismo celular necessitam de água para executarem suas atividades. Entretanto, se não realizada adequadamente, a secagem pode possibilitar a degradação de componentes químicos, facilitar o crescimento de microrganismos e comprometer o teor dos princípios ativos (CARVALHO et al., 2010; MARTINAZZO, 2006).

A armazenagem incorreta pode levar a perda de material, seja por motivos de ordem física ou biológica e quando bem conduzida possibilita manter os componentes do produto e evita a proliferação de microrganismos, parasitos, fungos e insetos. Falhas em qualquer uma dessas etapas influenciam diretamente na qualidade do produto final, fato evidenciado nesta revisão, onde a presença dos fungos Aspergillus sp., Penicillium sp., Fusarium sp. foi significativa nos produtos de origem vegetal (BARBOSA et al., 2010; MARTINAZZO, 2006).

Alguns agricultores e produtores desconhecem os cuidados necessários nas diversas etapas da cadeia produtiva para a obtenção de matérias-primas e produtos de qualidade adequada, outros julgam esses cuidados como desnecessários. A falta de condições sanitárias mínimas possibilita a contaminação ambiental por dejetos humanos que podem conter cistos, ovos e larvas de helmintos além de bactérias oriundas da microbiota intestinal, poluindo a água utilizada na irrigação das plantas. Na maioria dos casos, esses produtores, não contam com a orientação e acompanhamento de profissionais capacitados, o que pode justificar os altos níveis de contaminação encontrados (NERES et al., 2011).

Segundo Barbosa et al., (2010) parte das patologias causadas por produtos para o consumo humano é causada por microrganismos patogênicos transmitidos basicamente pela rota fecal-oral, ou seja, são excretados nas fezes de indivídu- os infectados e ingeridos na forma de água ou produto vegetal contaminado. Esses são denominados coliformes fecais, e são indicadores de baixas condições sanitárias, e incluem todas as bactérias aeróbicas ou anaeróbicas facultativas, gram-negativas, não esporuladas e na forma de bastonete, que fermentam a lactose com formação de gás dentro de $48 \mathrm{~h}$ a $35^{\circ} \mathrm{C}$ e nessa pesquisa merecem destaque, pois foram identificados em 6 dos 9 artigos avaliados.

Atenção especial deve ser dada ao fato de em um estudo ter sido constatado a presença de Salmonella sp., pertencente à família Enterobacteriaceae e responsável por intoxicações alimentares, a maioria dos sorotipos desse microrganismo é patogênica ao ser humano, apresentando diferenças de sintomatologia em decorrência dos diversos mecanismos de patogenicidade, idade do indivíduo infectado e da resposta imunológica (FRITTOLI; RODRIGUES, 2014).

Os parasitos encontrados em produtos de origem vegetal englobam diversas espécies de protozoários, helmintos e nematelmintos, cistos de Entamoeba histolystica, ovos de Ancilostomídeos e larvas filarióides de Ancilostomídeos associados à presença de Entamoeba coli e Endolimax nana, em amostras de alimentos são indicadores de baixas condições sanitárias que suscitam potencial contaminação fecal/ oral (FERNANDES et al., 2014; NERES et al., 2011).

Ascaris lumbricoides e Ancilostomídeos identificados em 7 dos 15 estudos, seguidos por Entamoeba coli e Estrogiloides estercolaris em 6 e 5 estudos respectivamente, Giardia lamblia e Taenia sp. aparecem em 3 estudos. Dados estes que conferem com contaminação fecal/oral. O tema contaminação por enteroparasitos, apesar de recorrente na literatura, não apresentou trabalhos que avaliaram a contaminação por estes em plantas medicinais, sendo a contaminação em hortaliças utilizada na pesquisa devido à semelhança na forma de cultivo e consumo e os benefícios para o conhecimento do potencial de contaminação por parasitos em plantas medicinais.

Parasitos intestinais são um problema de saúde pública no Brasil e em países em desenvolvimento, infecções parasitárias intestinais são comuns na infância, acometendo principalmente nas populações de classes socioeconômicas baixas, devido à falta de saneamento básico. Estes podem desencadear uma série de problemas no indivíduo infectado como alterações de humor, ansiedade e agitação. A. lumbricoides, $T$. trichura e G. lamblia podem ocasionar infecções assintomáticas e em quadros mais graves até síndrome da má 
absorção seguida de emagrecimento e o desenvolvimento de anemias, quadros de diarreia, oclusão, perfuração intestinal e dores abdominais. Em crianças parasitadas o comprometimento do desenvolvimento físico, com alterações no crescimento e desenvolvimento motor, intelectual com um rendimento diminuído nas atividades escolares além do déficit imunitário que as parasitoses intestinais podem gerar é preocupante (MATA-SANTOS et al., 2013; NETTO; GORETH DE SOUZA BRITO; PAVANELLI, 2016).

Os microrganismos comumente encontrados em plantas contaminadas são bactérias formadoras de esporos como Bacillus cereus e Clostridium perfringens, embora outros patógenos como Escherichia coli e Salmonella estejam muitas vezes presentes. Bolores causados por Fusarium spp., Aspergillus spp. e Penicillium spp. também podem ser encontrados. Contagens elevadas de fungos, em virtude da possibilidade de causarem intoxicações, constituem um risco por si só ou por serem produtores de micotoxinas, como a aflatoxina. Além disso, Staphylococcus aureus e Psedomonas aeruginosa são indicadores de contaminação diversificada (CARVALHO et al., 2010; TAKAYANAGUI et al., 2001).

No Brasil, há uma escassez de trabalhos avaliando a qualidade das plantas medicinais consumidas pela população, entretanto, visto que é um país que apresenta condições de saneamento precárias, alimentos "in natura", de um modo geral, podem estar contaminados. Isso demonstra a necessidade da conscientização por parte dos agricultores no sentido de se adequarem às boas práticas de cultivo de plantas medicinais, em especial no caso de plantas pilosas e rasteiras a fim de assegurar a qualidade microbiológica e parasitológica das mesmas (BOCHNER et al., 2012; BRASIL. MINISTÉRIO DA SAÚDE, 2006; CARNEIRO et al., 2014; LANINI et al., 2009; NERES et al., 2011).

\section{Conclusão}

Pesquisas que avaliaram a potencial contaminação microbiológica em plantas medicinais são escassas e no período e bases científicas pesquisadas nenhum trabalho avaliou a contaminação parasitológica dessas plantas, o que ressalta a importância do desenvolvimento de novas pesquisas envolvendo a avaliação do controle de qualidade microbiológico e parasitológico das plantas medicinais ofertadas a população. A falta de fiscalização e controle, fácil acesso, o risco de contaminação e as características dos usuários são fatores de risco para o desenvolvimento de problemas em decorrência do uso de plantas medicinais de baixa qualidade como opção terapêutica.

Estudos regionais a fim de determinar a presença de parasitos e microrganismos nos produtos de origem vegetal se apresentam como uma estratégia para avaliar as condições de higiene e o potencial de transmissão de parasitos intestinais através desses produtos, corrigindo pontos problemáticos na cadeia produtiva e evitando doenças veiculadas por meio da ingesta de produtos de origem vegetal contaminados através da rota fecal/oral.

\section{Referências}

ANDRADE, E. C. de. et al. Parasitoses intestinais : uma revisão sobre seus aspectos sociais, clínicos e terapêuticoS.
Revista APS, v. 13, n. 2, p. 231-240, 2010.

ARBOS, K. A. et al. Segurança alimentar de hortaliças orgânicas: aspectos sanitários e nutricionais. Ciência e Tecnologia de Alimentos, v. 30, n. 1, p. 215-220, 2010.

BARBOSA, C. K. R. et al. Qualidade Microbiológica De Plantas Medicinais Cultivadas Em Hortas Domésticas.

Revista Biotemas, v. 16, n. 2, p. 133-138, 2010.

BELINELO, V. J. et al. Enteroparasitas em hortaliças comercializadas na cidade de São Mateus, ES, Brasil. Arquivos de Ciência da Saúde Unipar, Umuarama, v. 13, n. 1, p. 33-36, 2009.

BEVILAQUA, G. A. P. et al. Plantas Bioativas em. Embrapa Clima Temperado. Documentos, 347, p. 26 p., 2012.

BEZERRA CARVALHO, A. C. et al. Regulation of herbal medicines in Brazil. Journal of Ethnopharmacology, v. 2013, n. PART B, p. 503-506, 2014.

BOCHNER, R. et al. Problems associated with the use of medicinal plants commercialized in "Mercadão de Madureira", Rio de Janeiro City, Brazil. Revista Brasileira de Plantas Medicinais, v. 14, n. 3, p. 537-547, 2012.

BRASIL. MINISTÉRIO DA SAÚDE. Portaria n 971, de 03 de maio de 2006, 2006. Disponível em: $<$ http://bvsms. saude.gov.br/bvs/saudelegis/gm/2006/prt0971_03_05_2006. html>

CARNEIRO, F. M. et al. Tendências Dos Estudos Com Plantas Medicinais No Brasil. Revista Sapiência: sociedade, saberes e práticas educacionais, v. 3 , n. 2 , p. 44-75, 2014.

CARVALHO, L. M. de. et al. Qualidade em plantas medicinais. Embrapa Tabuleiros Costeiros, v. 162, p. 1-56, 2010.

COELHO, L. M. et al. Detecção de formas transmissíveis de enteroparasitas na água e nas hortaliças consumidas em comunidades escolares de Sorocaba, São Paulo, Brasil.

Revista da Sociedade Brasileira de Medicina Tropical, v. 34, n. 5, p. 479-482, 2001.

COSSATIS, N. de A. FUNDAÇÃO OSWALDO CRUZ. QUALIDADE MICROBIOLÓGICA E VIGILÂNCIA SANITÁRIA Rio de Janeiro. Dissertação apresentada ao Programa de Pós- Graduação em Vigilância Sanitária do Instituto Nacional de Controle de Qualidade em Saúde da Fundação Oswaldo Cruz, 2015.

DA SILVA ALVES, A.; DA CUNHA NETO, A.; ROSSIGNOLI, P. A. PARASITOS EM ALFACE-CRESPA (Lactuca sativa L.), DE PLANTIO CONVENCIONAL, COMERCIALIZADA EM SUPERMERCADOS DE CUIABÁ. Revista de Patologia Tropical, v. 42, n. 2, p. 217-229, 2013. 
DUEDU, K. O. et al. A comparative survey of the prevalence of human parasites found in fresh vegetables sold in supermarkets and open-aired markets in Accra, Ghana. BMC Research Notes, v. 7, n. 1, p. 836, 2014.

FERNANDES, K. C. et al. Contaminação por enteroparasitos em verduras de supermercados e feiras de umuarama - pr. Arq. Ciênc. Vet. Zool. UNIPAR, v. 17, n. 2, p. 115-119, 2014

FERRO, J. J. B.; COSTA-CRUZ, J. M.; BARCELOS, I. S. DA C. AVALIAÇÃO PARASITOLÓGICA DE ALFACES ( Lactuca sativa ) COMERCIALIZADAS NO MUNICÍPIO DE TANGARÁ DA SERRA, MATO GROSSO, BRASIL. Revista de Patologia Tropical, v. 41, n. 1, p. 47-54, 2012.

FRIAS, A. A. T.; SILVA, J. B. DA; TOZATO, H. D. C. Ocorrência de ovos de helmintos em hortaliças comercializadas na cidade de Apucarana (PR). Semina: Ciências Biológicas e da Saúde, v. 33, n. 1, p. 35-42, 2012.

FRITTOLI, R. B.; RODRIGUES, L. H. Analysis of Fecal Coliform and Salmonella Sp . in Samples Minimally. Revista Científica da FHO|UNIARARAS v., v. 2, n. 2, p. 14-20, 2014.

GONÇALVES, R. M.; DA SILVA, S. R. P.; STOBBE, N. S. FREQUENNCIA DE PARASITOS EM ALFACES (Lactuca sativa) CONSUMIDAS EM RESTAURANTES self-service DE PORTO ALEGRE, RIO GRANDE DO SUL, BRASIL. Revista de Patologia Tropical, v. 42, n. 3, p. 323-330, 2013.

KŁAPEĆ, T.; BORECKA, A. Contamination of soil with geohelminth eggs on vegetable organic farms in the Lublin voivodeship, Poland. Wiadomosci parazytologiczne, v. 55, n. 4, p. 405-409, 2009.

LANINI, J. et al. "O que vêm da terra não faz mal" - relatos de problemas relacionados ao uso de plantas medicinais por raizeiros de Diadema/SP. Brazilian Journal of Pharmacognosy, v. 19, n. 1 A, p. 121-129, 2009.

MARCONDES, N. S. P.; ESMERINO, L. A. Qualidade Microbiológica De Plantas Medicinais Cultivadas Em Hortas Domésticas. Publicatio UEPG: Ciencias Biologicas e da Saude, v. 16, n. 2, p. 133-138, 2010.

MARTINAZZO, A. P. Secagem, armazenamento e qualidade de folhas de Cymbopogon citratus (D.C.) Stapf. Tese, Universidade Federal de Viçosa, p. 140, 2006.

MATA-SANTOS, T. et al. Prevalência de enteroparasitas em crianças atendidas em unidades básicas de saúde em uma cidade do sul do Brasil Prevalence of enteroparasites in children attended at basic health unities in a Brazilian southern city. Rev Inst Adolfo Lutz., v. 72, n. 2, p. 175178,2013

MAXIMINO, F. L. et al. Avaliação da descontaminação fúngica de camomila [Chamomilla recutita (L.) Rauschert] por meio de diferentes métodos caseiros em duas temperaturas. Revista Brasileira de Plantas Medicinais, v. 13, n. 4, p. 396-400, 2011.

MELO, J. G. DE et al. Qualidade de produtos a base de plantas medicinais comercializados no Brasil: castanhada-índia (Aesculus hippocastanum L.), capim-limão (Cymbopogon citratus (DC.) Stapf) e centela (Centella asiatica (L.) Urban). Acta Botanica Brasilica, v. 21, n. 1, p. 27-36, 2007.

NERES, A. C. et al. INTESTINAL PARASITES IN SAMPLES OF LETTUCE (Lactuva sativa var. crispa) FROM THE MUNICIPALITY OF ANAPOLIS, STATE OF GOIAS, BRAZIL. Bioscience Journal, v. 27, n. 2, p. 336-341, 2011.

NETTO, A. M.; GORETH DE SOUZA BRITO, M.; PAVANELLI, M. F. Relação entre enteroparasitoses e alterações hematológicas em crianças da região centrooeste do Paraná Relationship between enteroparasitosis and hematological changes in children in midwest of Paraná. Rbac, v. 48, n. 1, p. 78-84, 2016.

NOMURA, P. R. et al. Estudo da incidência de parasitas intestinais em verduras comercializadas em feira livre e supermercado de Londrina. Semina: Ciências Biológicas e da Saúde, Londrina, v. v. 36, n. n. 1, p. 209-214, 2015.

PACIFICO, B. B.; BASTOS, O. M. P.; UCHÔA, C. M. A. Contaminação parasitária em alfaces crespas (Lactuca sativa var. crispa ), de cultivos tradicional e hidropônico , comercializadas em feiras livres do Rio de Janeiro ( RJ ). Revista Instituto Adolfo Lutz, v. 72, n. 3, p. 219-225, 2013.

PAULA, P. et al. Contaminação microbiológica e parasitológica em alfaces (Lactuca sativa) de restaurantes self-service, de Niterói, RJ. Revista da Sociedade Brasileira de Medicina Tropical, v. 36, n. 4, p. 535-537, 2003.

RATAJCZAK, M. et al. Microbiological quality of nonsterile pharmaceutical products. Saudi Pharmaceutical Journal, v. 23, n. 3, p. 303-307, 2015.

ROSTAMI, A. et al. Contamination of commonly consumed raw vegetables with soil transmitted helminth eggs in Mazandaran province, northern Iran. International Journal of Food Microbiology, v. 225, p. 54-58, 2016.

SANTOS, R. L. et al. Contaminação fúngica de plantas medicinais utilizadas em chás. Revista de Ciencias Farmaceuticas Basica e Aplicada, v. 34, n. 2, p. 289-293, 2013.

SCHEFFER, M. C. et al. Boas Práticas Agrícolas (BPA) de plantas medicinais, aromáticas e condimentaresMinistério da Agricultura, Pecuária e Abastecimento Secretaria de Desenvolvimento 
Agropecuário e CooperativismoBrasília, DF, 2006.

SILVA, J. D. C. N. P. L. L. M. et al. ANÁLISE

MICROBIOLÓGICA DE ERVAS MEDICINAIS NA

CIDADE DE VITÓRIA DE SANTO ANTÃO - PE

JULIANA. Anais da Academia Pernambucana de

Ciência Agronômica, v. vols. 8 e, p. 205-216, 2012.

SOARES, B.; CANTOS, G. Qualidade parasitológica e condições higiênico-sanitárias de Florianópolis, Santa Catarina, Brasil. Revista Brasileira de Epidemiologia, v. 8, n. 4, p. 377-384, 2005.

SOUSA, E. R. D. E. Segurança microbiológica de plantas medicinais. [s.1.] Universidade Federal de Lavras, 2014.

SOUZA, T. P. DE; LIONZO, M. I. Z.; PETROVICK, P. R. Avaliação da redução da carga microbiana de droga vegetal através do processamento tecnológico: decocção e secagem por aspersão. Revista Brasileira de Farmacognosia, v. 16, n. 1, p. 94-98, 2006.

TAKAYANAGUI, O. M. et al. Fiscalização de verduras comercializadas no município de Ribeirão Preto, SP Monitoring of vegetables commercially sold in Ribeirão Preto, SP, Brazil. Revista Brasileira De Medicina Tropical, v. 34, n. 1, p. 37-41, 2001.

YESUF, A. et al. Occurrence of Potential Bacterial Pathogens and Their Antimicrobial Susceptibility Patterns Isolated from Herbal Medicinal Products Sold in Different Markets of Gondar Town, Northwest Ethiopia. International journal of bacteriology, v. 2016, p. 1959418, 2016.

ZARONI, M. et al. Qualidade microbiológica das plantas medicinais produzidas no Estado do Paraná. Revista

Brasileira de Farmacognosia, v. 14, n. 1, p. 29-39, 2004. 